\title{
Effects of transcription factor EB on oxidative stress and apoptosis induced by high glucose in podocytes
}

\author{
YINGLI KANG, YING LI, TAO ZHANG, YANQING CHI and MAODONG LIU \\ Department of Nephrology, The Third Hospital of Hebei Medical University, \\ Shijiazhuang, Hebei 050051, P.R. China
}

Received September 29, 2018; Accepted May 20, 2019

DOI: $10.3892 /$ ijmm.2019.4209

\begin{abstract}
The aim of the present study was to investigate the effects of transcription factor EB (TFEB) overexpression on oxidative stress, mitochondrial function and apoptosis in podocytes induced with high glucose. High glucose-induced time-dependent changes in TFEB expression were identified and nuclear translocation of TFEB was observed in podocytes. Overexpression of TFEB markedly reduced high glucose-induced oxidative stress in podocytes, and increased the expression of superoxide dismutase 2 and heme oxygenase 1 antioxidant enzymes. It was further observed that TFEB overexpression could partially restore the expression of peroxisome proliferator-activated receptor- $\gamma$ coactivator- $1 \alpha$, transcription factor $\mathrm{A}$, mitochondrial, and cytochrome $c$ oxidase subunit 4, thereby enhancing mitochondrial biosynthesis. Furthermore, overexpression of TFEB reduced mitochondrial swelling and fragmentation, restored mitochondrial membrane potential, and contributed to the restoration of mitochondrial function. By overexpressing TFEB, it was revealed that TFEB increased the ratios of phosphorylated (p)-Akt/Akt and p-Bad/Bad, and the expression of downstream Bcl-xl, and reduced the ratio of Bax/Bcl-2 and the expression of cleaved-caspase-3 compared with high glucose-treatment. Furthermore, when the Akt phosphorylation inhibitor Ly294002 was added, the improvement by TFEB to high glucose-induced apoptosis was significantly reduced. These findings suggest that overexpressing TFEB could reduce the production of reactive oxygen species in podocytes in a high glucose environment, relieve oxidative stress, promote mitochondrial biogenesis and renewal functions, and reduce high glucose-induced podocyte apoptosis by activating the Akt/Bad pathway.
\end{abstract}

Correspondence to: Professor Ying Li, Department of Nephrology, The Third Hospital of Hebei Medical University, 102 Youyi North Street, Shijiazhuang, Hebei 050051, P.R. China

E-mail: liyinghebei@126.com

Key words: diabetic nephropathy, apoptosis, reactive oxygen species, oxidative stress, podocyte, transcription factor EB

\section{Introduction}

Diabetic nephropathy (DN) is a leading cause of end-stage kidney disease (1-3). Globally, diabetes has previously been reported to be associated with a substantially increased risk of mortality. However, this increased risk is predominantly identified in patients with both diabetes and kidney disease (1). The increasing incidence rates of diabetes and DN are a global public health problem and bring a heavy economic burden to society (3). Therefore, it is necessary to elucidate the pathogenesis and identify novel therapeutic targets of DN.

Podocytes serve a vital role in the glomerular filtration barrier (4). Glomerular filtration barrier injury is a hallmark of DN, and a notable cause of glomerulosclerosis and impaired renal function (5). Hyperglycemia can induce podocyte oxidative stress, apoptosis and inflammation (6). Reactive oxygen species (ROS) serve a vital role in the development of diabetes and diabetic complications (7). According to numerous studies, ROS are important secondary messengers for signaling pathways associated with apoptosis, proliferation, damage and inflammation (8-11). At the early stages of diabetes, podocyte apoptosis induced by hyperglycemia can cause glomerular hyperfiltration (12). High glucose (HG) can not only cause mitochondrial structural destruction and dysfunction by affecting mitochondrial biogenesis, mitophagy and mitochondrial dynamics, but can also release certain molecules that induce apoptosis. Therefore, reducing ROS production and blocking mitochondria-mediated podocyte apoptosis have potential significance in the treatment of diabetic nephropathy (13). However, the precise underlying molecular mechanism is not well understood.

The transcription factor EB (TFEB) is a member of the microphthalmia-transcription factor $E$ family of basic helix-loop-helix-leucine-zipper transcription factors (14). The role of TFEB as a 'master regulator' of the autophagy-lysosome pathway has been widely investigated $(15,16)$. In addition, TFEB is involved in a variety of pathophysiological processes, including modulating mitochondrial function through the peroxisome proliferator-activated receptor- $\gamma$ coactivator- $1 \alpha$ (PGC-1 $\alpha$ ) and phosphatase and tensin homolog-induced kinase pathways trigged by ROS $(17,18)$, responding to endoplasmic reticulum stress through calcium signaling (19), and participating in immune responses via macrophage cells (20). Advanced glycation end products have been demonstrated 
to reduce the expression of TFEB in glomerular mesangial cells and produce significant oxidative stress (21). In addition, monolayer podocytes overexpressing TFEB were able to restore selective permeability of albumin (22).

To the best of our knowledge, there has been limited investigation regarding the associations between TFEB and kidney diseases, particularly DN. The role of TFEB in the pathogenesis and progression of DN remains unknown. The present study hypothesized that decreased TFEB in DN may promote mitochondrial damage, excessive ROS production and subsequent podocyte apoptosis. The current study evaluated the changes in TFEB expression in HG cultured podocytes in vitro, and the effects of overexpressing TFEB on ROS production, oxidative stress, mitochondrial damage and apoptosis. Furthermore, possible molecular mechanisms were investigated.

\section{Materials and methods}

Cell culture. Conditioned immortalized mouse podocytes (cat. no. 3111C0001CCC000230) were purchased from the Basic Medical Cell Center of Beijing Union Medical College (http://www.cellresource.cn/). The present study was approved by the Pathology Laboratory of Hebei Medical University (Shijiazhuang, China) for the use of purchased mouse podocytes. Mouse podocytes were cultured in DMEM-F12 (glucose, 1 g/l; Gibco; Thermo Fisher Scientific, Inc.) supplemented with $10 \%$ fetal bovine serum (Biological Industries, Kibbutz Beit-Haemek, Israel) and $10 \mathrm{U} / \mathrm{ml}$ IFN- $\gamma$. Subsequently, the differentiation of podocytes was induced in the aforementioned medium without IFN- $\gamma$ at $37^{\circ} \mathrm{C}$ in a humidified atmosphere with $5 \% \mathrm{CO}_{2}$. Mature podocytes were used for subsequent experiments after $\sim 10$ days.

Stimulation at $37^{\circ} \mathrm{C}$ was initiated when the cells grew to $60-70 \%$, and the cells were divided into a normal glucose (NG) group $(5.5 \mathrm{mmol} / \mathrm{l} \mathrm{D}$-glucose; Sigma-Aldrich; Merck KGaA), a mannitol-hypertonic control group $(5.5 \mathrm{mmol} / \mathrm{l}$ D-glucose + 24.5 mmol/1 D-mannitol; Sigma-Aldrich; Merck $\mathrm{KGaA}$ ) and a HG group (30 mmol/1 D-glucose). Fresh medium was provided daily to ensure the stability of the glucose concentration. Preliminary experiments demonstrated that the stability of the glucose concentration could be maintained via providing fresh medium daily (data not shown). Previous studies have reported that it's possible to maintain a stable glucose concentration for $72 \mathrm{~h}$ (23-25). In addition, $\mathrm{N}$-acetylcysteine (NAC; $5 \mathrm{mmol} / \mathrm{l}$; MedChem Express) and Ly294002 (20 $\mu \mathrm{mol} / \mathrm{l}$; MedChem Express) were directly added to the HG group at $37^{\circ} \mathrm{C}$ for $48 \mathrm{~h}$.

Plasmids and transfection. When the cells were grown to 70-90\% confluence, $2.5 \mu \mathrm{g}$ pcDNA3.1 (+)-TFEB plasmid (Cyagen Biosciences, Inc.) or the control pcDNA3.1 (+)-vector (Cyagen Biosciences, Inc.) were transfected into different groups of mouse podocytes using Lipofectamine ${ }^{\circledR} 3000$ (Thermo Fisher Scientific Inc.), according to the manufacturer's protocol. Each well of a six-well plate contained $2.5 \mu \mathrm{g}$ plasmid or control vector, $3.75 \mu \mathrm{l}$ Lipofectamine 3000 reagent and $5 \mu \mathrm{l}$ P3000 reagent. The cells were cultured in an incubator at $37^{\circ} \mathrm{C}$ with $5 \% \mathrm{CO}_{2}$. Following $6 \mathrm{~h}$, fresh medium was provided as required. After $24 \mathrm{~h}$, the aforementioned stimulations were initiated for $48 \mathrm{~h}$.
Western blotting. Protein was extracted from the treated cells using RIPA lysate with proteinase inhibitor and phosphatase inhibitor (Roche Diagnostics) for $30 \mathrm{~min}$ on ice. The fractions of nuclear protein and cytoplasmic protein were separated using Minute ${ }^{\mathrm{TM}}$ Cytoplasmic and Nuclear Extraction kit (cat. no. SC-003; Invent Biotechnologies, Inc.), according to the manufacturer's protocol. The protein concentration was detected using the BCA method. Equal amounts of protein $(30 \mu \mathrm{g})$ were separated by $12 \%$ SDS-PAGE. Following electrophoresis, the targeted proteins on the gel were transferred to polyvinylidene difluoride (PVDF) membranes and blocked with $5 \%$ skim milk overnight at $4^{\circ} \mathrm{C}$. Subsequently, the membrane was incubated with primary antibodies at room temperature for $2 \mathrm{~h}$. After three washes with TBS and $0.5 \%$ Tween-20, the membrane was incubated with horseradish peroxidase-labeled secondary antibody (goat anti-rabbit; cat. no. 074-1506; 1:10,000; KPL, Inc.) for $1 \mathrm{~h}$ at room temperature. Subsequently, the PVDF membrane was immersed into an electrochemiluminescence solution (Tangen Biosciences, Inc.) for $2 \mathrm{~min}$ in a dark room. Finally, the band intensities were quantified using ImageJ 1.8.0 software (National Institutes of Health). The primary antibodies anti-Akt (cat. no. 4691; 1:1,000), anti-phosphorylated (p)-Akt (Thr308; cat. no. 9275; 1:1,000), anti-Bad (cat. no. 9239; 1:1,000) and anti-p-Bad (Ser112; cat. no. 5284; 1:1,000) were purchased from Cell Signaling Technology, Inc.. Anti-cleaved-caspase-3 (cat. no. 29034; 1:500) and anti-nephrin (cat. no. 31249; 1:1,000) were purchased from Signalway Antibody LLC. Anti-TFEB (cat. no. 13372-1-AP; 1:1,000), anti-superoxide dismutase 2 (SOD2; cat. no. 24127-1-AP; 1:2:000), anti-heme oxygenase 1 (HO1; cat. no. 10701-1-AP; 1:2,000), anti-PGC1 $\alpha$ (cat. no. 20658-1-AP; 1:1,000) anti-transcription factor mitochondrial (TFAM; cat. no. 19998-1-AP; 1:1,000) and anti-cytochrome c oxidase subunit IV (COX IV; cat. no. 11242-1-AP; 1:1,000) were purchased from Protein Tech Group, Inc.. Anti-Bcl-2 (cat. no. ab59348; 1:1,000), anti-Bax (cat. no. ab32503; 1:2,000) and anti-Bcl-xl (cat. no. ab32370; $1: 1,000)$ were purchased from Abcam. Anti- $\beta$-actin (cat. no. AC026: 1:10,000) was purchased from ABclonal Biotech Co., Ltd. and anti-lamin B1 (cat. no. ARG65740; 1:2,000) was purchased from Arigo Biolaboratories Corp.

Cell immunofluorescence. The cells $\left(1 \times 10^{6}\right)$ cultured in six-well plates were fixed in $4 \%$ paraformaldehyde at room temperature for $15 \mathrm{~min}$ and then treated with $0.2 \%$ Triton diluted in PBS for $10 \mathrm{~min}$. After washing with PBS, goat serum was used to block non-specific binding site at $37^{\circ} \mathrm{C}$ for $1 \mathrm{~h}$. Rabbit anti-mouse TFEB antibody (1:100) was then added and incubated overnight at $4^{\circ} \mathrm{C}$. Dylight 488 -labeled secondary antibody (cat. no. 072-03-15-06; 1:150; KPL, Inc.) was added and incubated at $37^{\circ} \mathrm{C}$ for $1 \mathrm{~h}$. After washing with PBS, the nuclei were stained with DAPI at room temperature for $5 \mathrm{~min}$. The cells were observed with a confocal microscope (magnification, x630; Leica Microsystems $\mathrm{GmbH}$ ).

Reverse transcription-quantitative polymerase chain reaction (RT-qPCR). RNA was extracted from the treated cell using TRIzol ${ }^{\circledR}$ reagent (Thermo Fisher Scientific Inc.). cDNA was synthesized using Reverse Transcription mix (Promega Corporation), followed by qPCR. The thermocycling conditions were as follows: $95^{\circ} \mathrm{C}$ for $60 \mathrm{sec}, 40$ cycles at $95^{\circ} \mathrm{C}$ for $5 \mathrm{sec}$, 
Table I. Primer sequences for reverse transcription-quantitative polymerase chain reaction.

\begin{tabular}{lll}
\hline Primer & \multicolumn{1}{c}{ Forward sequence } & \multicolumn{1}{c}{ Reverse sequence } \\
\hline TFEB & 5'-GTCTTGGGCAAATCCCTTCT-3' & 5'-TTCGGGCTCCCTGTAGTCG-3' \\
PGC-1 $\alpha$ & 5'-TGTGCTGCTCTGGTTGGT-3' & 5'-GTTGGATATGATTTCCGATT-3' \\
TFAM & 5'-GGAATGTGGAGCGTGCTA-3' & 5'-CAAGACTGATAGACGAGGG-3' \\
HO-1 & 5'-GCTGGTGATGGCTTCCTT-3' & 5'-GGGCATAGACTGGGTTCTG-3' \\
SOD-2 & 5'-GGGCATAGACTGGGTTCTG-3' & 5'-TTCTCCTCGGTGGCGTTG-3' \\
COX IV & 5'-TCACTGCGCTCGTTCTGAT-3' & 5'-CGATCGAAAGTATGAGGGATG-3' \\
$\beta$-actin & 5'-GGCTGTATTCCCCTCCATCG-3' & 5'-CCAGTTGGTAACAATGCCATGT-3'
\end{tabular}

TFEB, transcription factor EB; PGC-1 $\alpha$, peroxisome proliferator-activated receptor- $\gamma$ coactivator- $1 \alpha$; TFAM, transcription factor mitochondrial; HO-1, anti-heme oxygenase 1; SOD-2, superoxide dismutase 2; COX IV, cytochrome c oxidase subunit IV.

$56^{\circ} \mathrm{C}$ for $30 \mathrm{sec}$ and extension at $72^{\circ} \mathrm{C}$ for $30 \mathrm{sec}$. The mRNA levels of target genes were detected on an Agilent Mx3000P QPCR system (Agilent Technologies, Inc.). The relative quantities of mRNA were calculated using the $2^{-\Delta \Delta \mathrm{Cq}}$ method (26) and normalized to the housekeeping gene $\beta$-actin. The primer sequences for qPCR are presented in Table I.

Intracellular ROS detection. Cells $\left(1 \times 10^{5}\right)$ were cultured in six-well plates and washed three times with PBS following stimulation. Subsequently, $10 \mu \mathrm{mol} / 1$ fluorescent probe 2',7'-dichlorodihydrofluorescein diacetate (DCFH-DA) was added to all groups except the negative control group. The cells were then incubated in the dark for $30 \mathrm{~min}$ at $37^{\circ} \mathrm{C}$ and washed with PBS. Cells were collected into a flow-tube-specific tube with a filter and then analyzed using a flow cytometer (FACS-Aria II; BD Biosciences) within $1 \mathrm{~h}$ and FlowJo 7.6 (FlowJo LLC, Ashland, OR, USA) was used for analysis.

Mitochondrial ROS detection. After washing with pre-warmed Hanks' balanced salt solution/Ca/Mg buffer (Solarbio Science and Technology Co., Ltd.) three times, the cells were incubated in the dark with $5 \mu \mathrm{mol} / 1$ MitoSOX reagent (Thermo Fisher Scientific, Inc.) working solution at $37^{\circ} \mathrm{C}$ for $20 \mathrm{~min}$. The cells were observed immediately under a confocal microscope (magnification, x630) after being washed twice with Hanks' buffer.

Mitochondrial morphology detection. Following washing with pre-warmed PBS three times, the cells were incubated in the dark with $100 \mathrm{nmol} / 1$ MitoTracker Red working solution (Thermo Fisher Scientific Inc.) at $37^{\circ} \mathrm{C}$ for $15 \mathrm{~min}$. Following incubation, the cells were washed three times and then directly visualized by laser scanning confocal microscopy (magnification, x630; Leica Microsystems GmbH).

Detection of mitochondrial membrane potential. The cells $\left(1 \times 10^{6}\right)$ were cultured in a $60-\mathrm{mm}$ dish. Following stimulation, the cells were washed three times with PBS and $10 \mu \mathrm{g} / \mathrm{ml}$ JC-1 was added, according to the manufacturer's protocol. The cells were incubated in the dark at $37^{\circ} \mathrm{C}$ for $20 \mathrm{~min}$. After incubation, the cells were washed three times and immediately images were captured with a confocal microscope (magnification, x630; Leica Microsystems $\mathrm{GmbH}$ ). The change in mitochondrial membrane potential is represented by the ratio of red to green fluorescence.

Flow cytometry detection of cell apoptosis. Treated cells $\left(1 \times 10^{5}\right)$ were collected into an EP tube and washed twice with pre-chilled PBS. Subsequently, $100 \mu 1 \mathrm{IX}$ binding buffer (cat. no. 51-66121E; BD Pharmingen; BD Biosciences), $5 \mu$ PE Annexin V (cat. no. 51-65875X' BD Pharmingen; BD Biosciences) and $5 \mu 1$ 7-ADD (cat. no. 51-68981E; BD Pharmingen; BD Biosciences) were added according to the protocol, and the cells were incubated in the dark for $15 \mathrm{~min}$ at room temperature. Then, $400 \mu 1 \mathrm{X}$ binding buffer was added to each tube and transferred to a special flow tube with a filter protected from light. Cell apoptosis was detected using a flow cytometer (FACS-Aria II; BD Biosciences) within $1 \mathrm{~h}$ and FlowJo 7.6 (FlowJo LLC) was used for analysis.

Statistical analysis. SPSS 21.0 (IBM Corp.) was used to analyze all data. Data are presented as the mean \pm standard deviation. Each set of data represents at least three independent experiments. Differences among multiple groups were statistically analyzed using one-way ANOVA followed by Bonferroni's post hoc test. $\mathrm{P}<0.05$ was considered to indicate a statistically significant difference.

\section{Results}

$H G$ induces time-dependent changes in TFEB expression and induces its nuclear translocation in podocytes. The mRNA and protein levels of TFEB were detected by RT-qPCR and western blot analysis, respectively. The results demonstrated that the expression of TFEB induced by HG was time-dependent, with a tendency to increase first and then decrease. Compared with the NG group, the level of TFEB significantly increased after $6 \mathrm{~h}$ of HG culture and then gradually decreased. After $72 \mathrm{~h}$ of HG culture, the protein level of TFEB decreased to $58 \%$ of the level of the NG group and the mRNA level of TFEB decreased to $46 \%$ (Fig. 1A and B). However, no significant difference was observed in the expression level of TFEB between the NG group and the mannitol-hypertonic group. Within $24 \mathrm{~h}$ following HG stimulation, the TFEB level in the nucleus was significantly higher compared with that in the NG group, while the protein level of TFEB in the cytosol did not change 

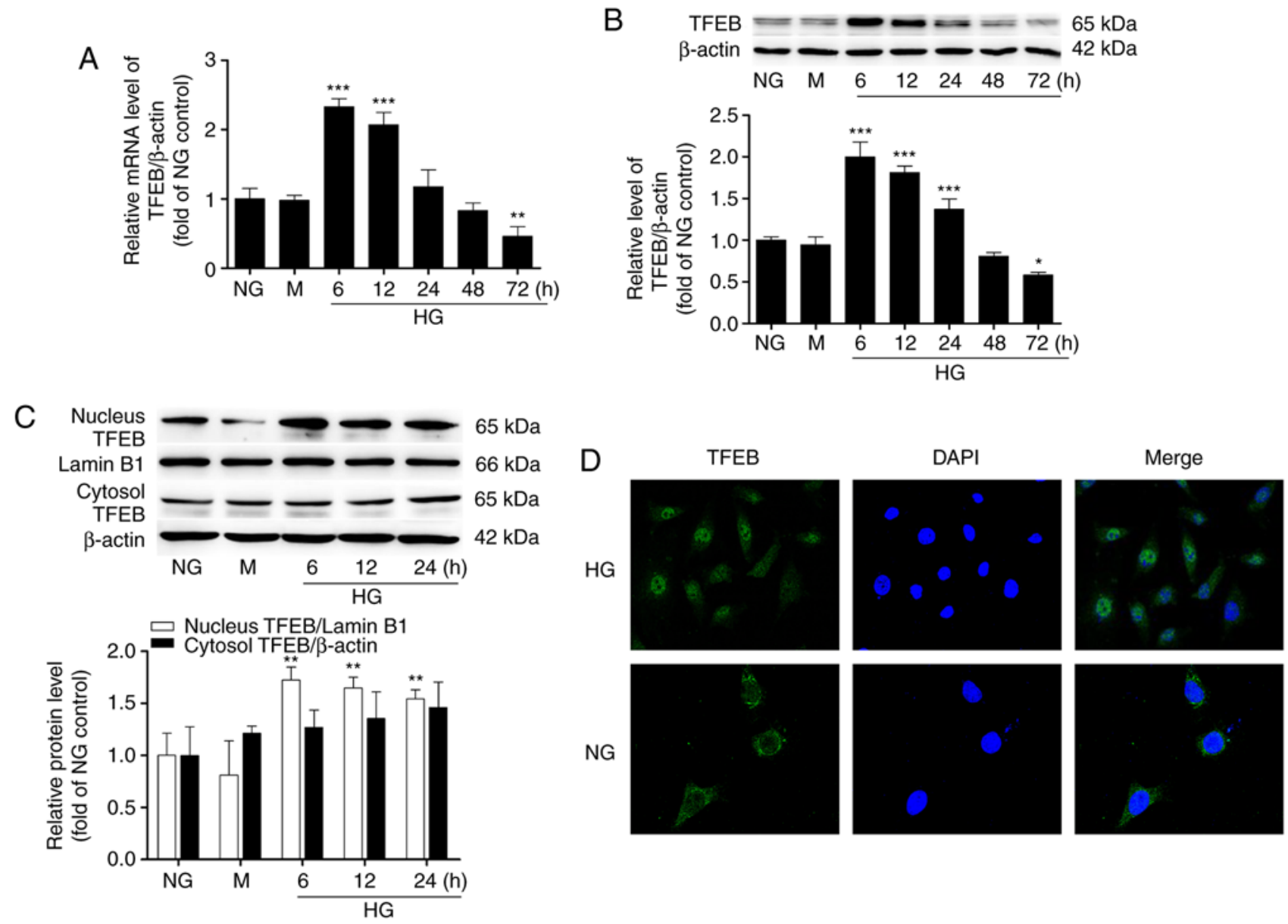

Figure 1. HG induces time-dependent changes in TFEB mRNA and protein expression and causes nuclear translocation. The (A) mRNA and (B) protein levels of TFEB in podocytes with different HG-stimulation times were analyzed by western blot and reverse transcription-quantitative polymerase chain reaction. (C) The protein levels of TFEB in the nucleus and cytosol of podocytes with different HG-stimulation times were detected by western blot analysis. (D) The distribution of TFEB (green) at $48 \mathrm{~h}$ after NG and HG was examined by confocal microscopy. Magnification, $x 630 .{ }^{*} \mathrm{P}<0.05,{ }^{* *} \mathrm{P}<0.01,{ }^{* * * *} \mathrm{P}<0.001 \mathrm{vs}$. NG. HG, high glucose; TFEB, transcription factor EB; NG, normal glucose; M, mannitol-hypertonic control.

significantly (Fig. 1C). Cellular immunofluorescence also revealed that the nuclear location of TFEB was weak and the cytoplasmic fluorescence was strong in the NG group, while the nuclear location of TFEB increased and the fluorescence of the nucleus was enhanced in the HG group (Fig. 1D).

Overexpression of TFEB alleviates $H G$-induced oxidative stress in mouse podocytes. To further clarify the role of TFEB in HG stimulation, plasmids overexpressing TFEB were transfected into mouse podocytes. The efficacy of transfection is presented in Fig. S1. It was identified that the expression of TFEB significantly increased following transfection with TFEB-overexpression plasmids. The present study further detected the expression of the anti-oxidative stress-related proteins SOD2 and HO1 using RT-qPCR and western blot analysis after $48 \mathrm{~h}$ of $\mathrm{HG}$ induction. The results demonstrated that the mRNA and protein levels of SOD2 and HO1 in the HG group were significantly decreased compared with those of the NG group. Overexpression of TFEB significantly reversed the HG-induced decease in SOD2 and HO1, and there was no significant difference from the positive control HG + NAC group. No significant changes were observed between the HG + control group (podocytes transfected with empty vectors) and the HG group (Fig. 2A and B).
Subsequently, the fluorescence probes MitoSOX Red and DCFH-DA were used to detect ROS in each group. The overexpression of TFEB and NAC-treatment significantly reduced the production and accumulation of ROS induced by HG. These results suggest that overexpression of TFEB could reduce the oxidative stress of podocytes induced by HG (Fig. 2C and D).

Overexpression of TFEB protects the morphology and function of podocyte mitochondria. Compared with the NG group, the expression levels of PGC- $1 \alpha$, TFAM and COX IV in the HG group were decreased by 44,41 and $40 \%$, respectively, while the mRNA and protein levels of PGC-1 $\alpha$, TFAM and COX IV were significantly increased following overexpression of TFEB (Fig. 3A and B), suggesting that TFEB enhanced the mitochondrial biogenesis function.

The summarized data for the number of normal/polarized mitochondria are presented in Fig. 3C. The JC-1 probe demonstrated that JC-1 red fluorescence in the NG group was significantly stronger compared with $\mathrm{JC}-1$ green. Compared with the NG group, JC-1 green fluorescence was significantly increased and mitochondrial membrane potential was significantly decreased in the HG group. The 


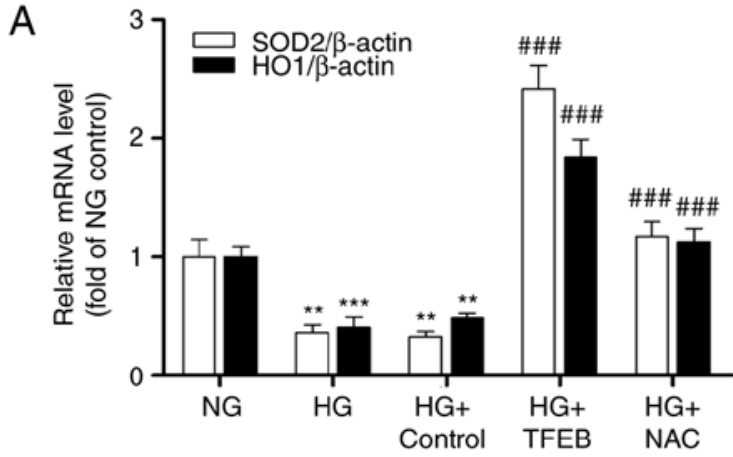

B
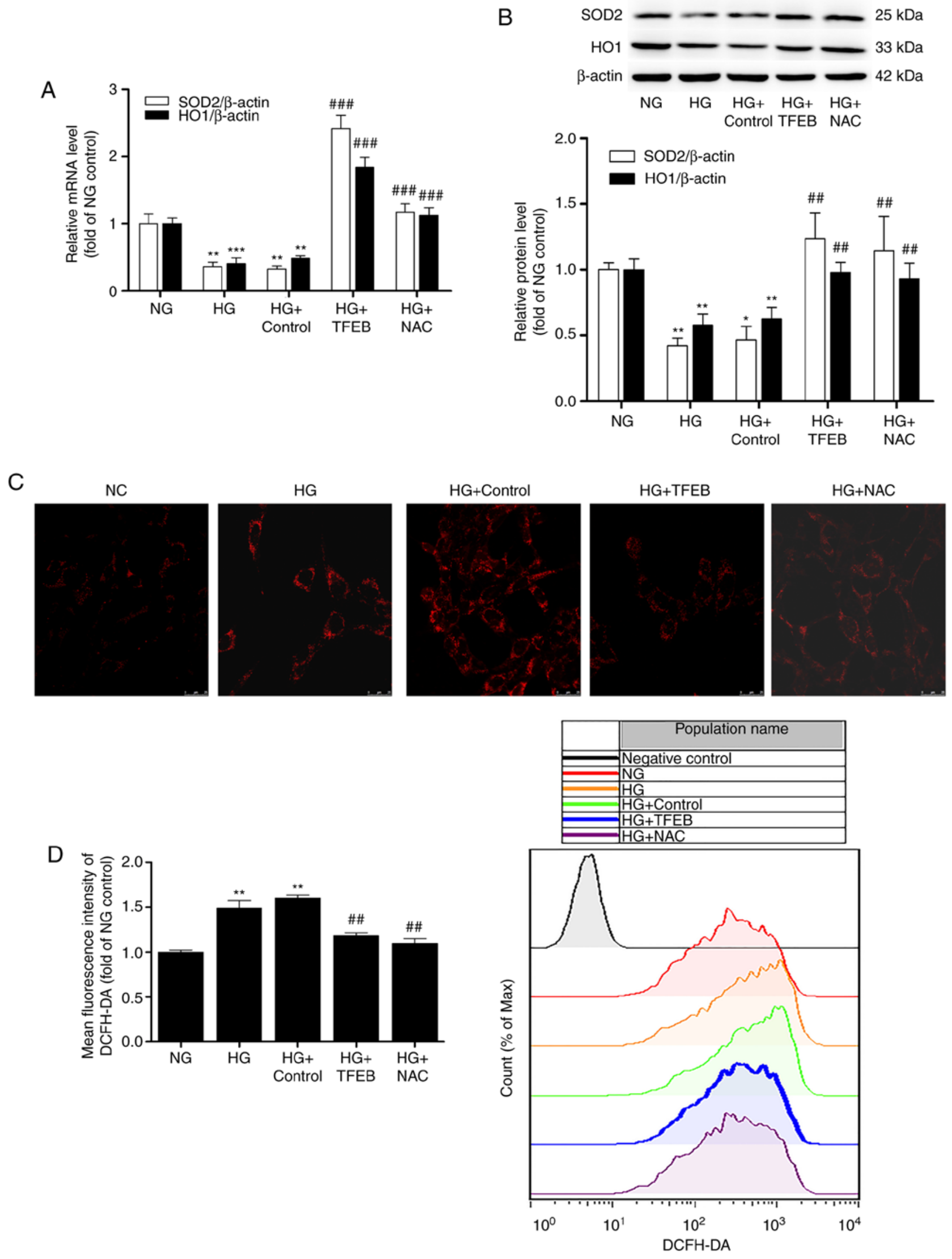

Figure 2. Overexpression of TFEB reduces HG-induced podocyte oxidative stress. The HG + TFEB group transfected with the TFEB expression vector and the $\mathrm{HG}+$ control group transfected with an empty plasmid vector were cultured in HG medium for $48 \mathrm{~h}$. Subsequently, (A) mRNA and (B) protein levels of SOD2 and HO1 were detected by reverse transcription-quantitative polymerase chain reaction and western blot analysis, respectively. (C) Detection of mitochondrial ROS accumulation by confocal microscopy. (D) Flow cytometry analysis of intracellular ROS using a DCFH-DA fluorescent probe. Scale bar=25 $\mu \mathrm{m}$; Magnification, $\mathrm{x} 630 .{ }^{*} \mathrm{P}<0.05,{ }^{* *} \mathrm{P}<0.01,{ }^{* * *} \mathrm{P}<0.001$ vs. NG group. ${ }^{\# \#} \mathrm{P}<0.01,{ }^{\# \# "} \mathrm{P}<0.001$ vs. $\mathrm{HG}+$ control group. HG, high glucose; TFEB, transcription factor EB; NG, normal glucose; NAC, N-acetylcysteine; SOD2, superoxide dismutase 2; HO1, anti-heme oxygenase 1; DCFH-DA, 2',7'-dichlorodihydrofluorescein diacetate. 


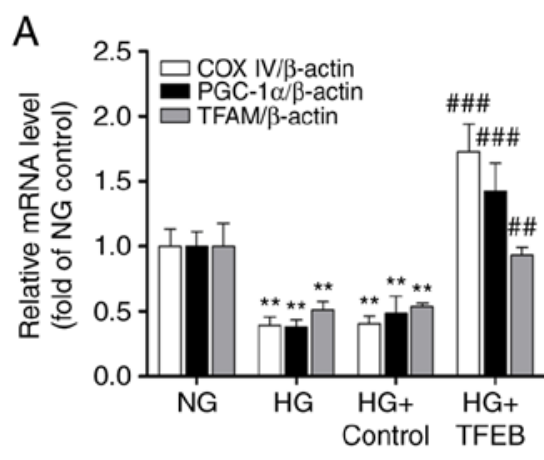

D

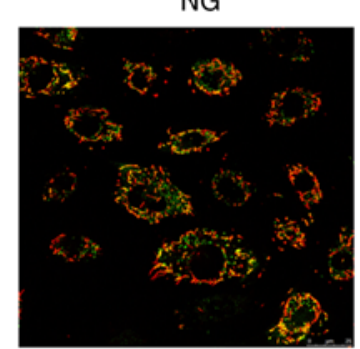

E

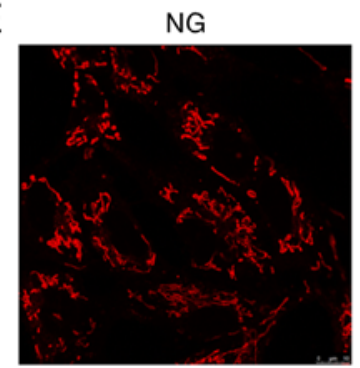

B $\operatorname{cox} I V-\longrightarrow-18 \mathrm{kDa}$

PGC-1 $1 \alpha---91 \mathrm{kDa}$

TFAM - - - $29 \mathrm{kDa}$

$\beta$-actin $-\overline{\mathrm{NG}}-\overline{\mathrm{HG}}-\underset{\mathrm{HG}+}{-}-\frac{\mathrm{HG}+}{\mathrm{kDa}} \mathrm{C}$

Control TFEB

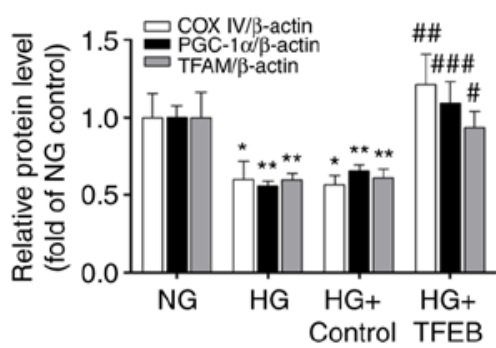

$\mathrm{HG}$

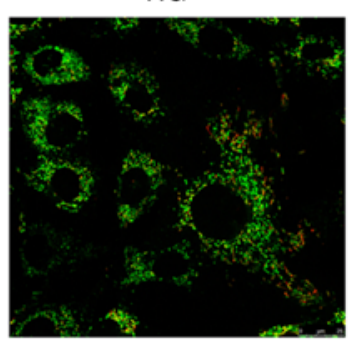

$H G$

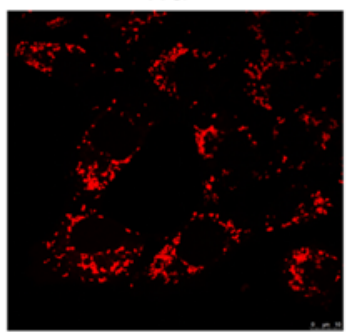

$\mathrm{HG}+$ Control

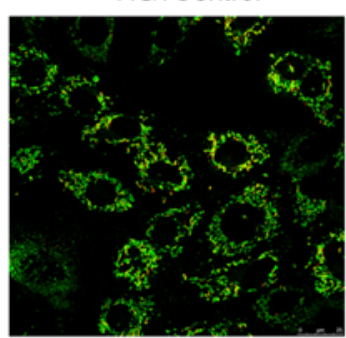

$\mathrm{HG}+\mathrm{Control}$

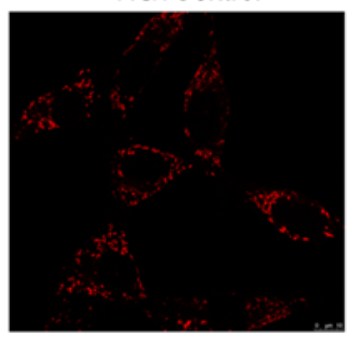

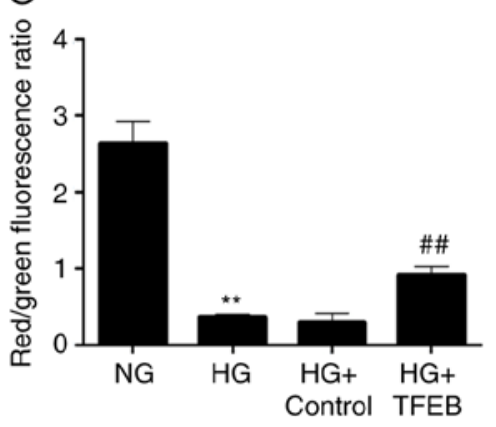

$\mathrm{HG}+\mathrm{TFEB}$
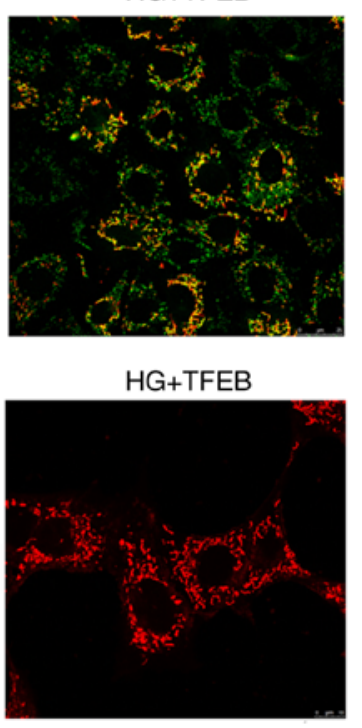

Figure 3. Overexpression of TFEB can maintain mitochondrial morphology and function. The HG + TFEB group transfected with the TFEB expression vector and the $\mathrm{HG}+$ control group transfected with an empty plasmid vector were cultured in HG medium for 48 h. Then, (A) mRNA and (B) protein levels of PGC-1 $\alpha$, TFAM and COX IV were detected by reverse transcription-quantitative polymerase chain reaction and western blot analysis. (C) The red fluorescence/green fluorescence ratio indicates the change in mitochondrial membrane potential. (D) Detection of mitochondrial morphology by confocal microscopy. Scale bar $=10 \mu \mathrm{m}$; Magnification, x630. (E) Change of mitochondrial membrane potential of podocytes detected by confocal microscopy. Scale bar=25 $\mu \mathrm{m}$; Magnification, $\mathrm{x} 630$. ${ }^{*} \mathrm{P}<0.05,{ }^{* *} \mathrm{P}<0.01$ vs. NG group. ${ }^{\#} \mathrm{P}<0.05,{ }^{\#} \mathrm{P}<0.01,{ }^{\# \# \#} \mathrm{P}<0.001$ vs. HG $+\mathrm{Control}$ group. TFEB, transcription factor EB; $\mathrm{HG}$, high glucose; PGC-1 $\alpha$, peroxisome proliferator-activated receptor- $\gamma$ coactivator-1 $\alpha$; TFAM, transcription factor mitochondrial; COX IV, cytochrome c oxidase subunit IV; NG, normal glucose.

TFEB-overexpression group demonstrated partial recovery of the membrane potential reduced by $\mathrm{HG}$ and an increase in the number of polarized mitochondria (Fig. 3D). Under the confocal microscope, the MitoTracker Red probe revealed that the mitochondria in the HG group demonstrated swelling and fragmentation compared with those in the NG group. Compared with the HG group, the TFEB-overexpression group demonstrated reduced mitochondrial fragmentation and the number of mitochondria with normal morphology increased (Fig. 3E).

Overexpression of TFEB alleviates $H G$-induced podocyte apoptosis by activating the Akt/Bad pathway. With prolonged HG stimulation, the expression of nephrin gradually decreased, while cleaved-caspase-3 and Bax/Bcl-2 gradually increased. By 48 h, nephrin decreased by $40 \%$ in the HG group compared with the NG group, while cleaved-caspase-3 and Bax/Bcl-2 demonstrated a 1.23 and 2.35 -fold increase, respectively (Fig. 4A). Furthermore, the phosphorylation levels of Akt and Bad were altered following HG stimulation. The level of p-Akt first increased and then decreased; the p-Akt/Akt ratio increased by $70 \%$ after $12 \mathrm{~h}$ of $\mathrm{HG}$ stimulation, and the p-Akt/Akt and p-Bad/Bad ratios decreased to 48 and $45 \%$ of the NG group after $72 \mathrm{~h}$ of $\mathrm{HG}$ stimulation, respectively (Fig. 4B).

By overexpressing TFEB, it was observed that compared with HG, TFEB significantly increased the ratios of p-Akt/Akt and $\mathrm{p}-\mathrm{Bad} / \mathrm{Bad}$, significantly enhanced the expression of downstream Bcl-xl, significantly reduced the ratio of Bax/Bcl-2, significantly reduced the expression of cleaved-caspase- 3 and significantly increased the expression of nephrin. Furthermore, when Ly294002 was added, p-Akt, p-Bad, Bcl-xl and nephrin were significantly inhibited compared with the HG + TFEB group (Fig. 4C and D). 

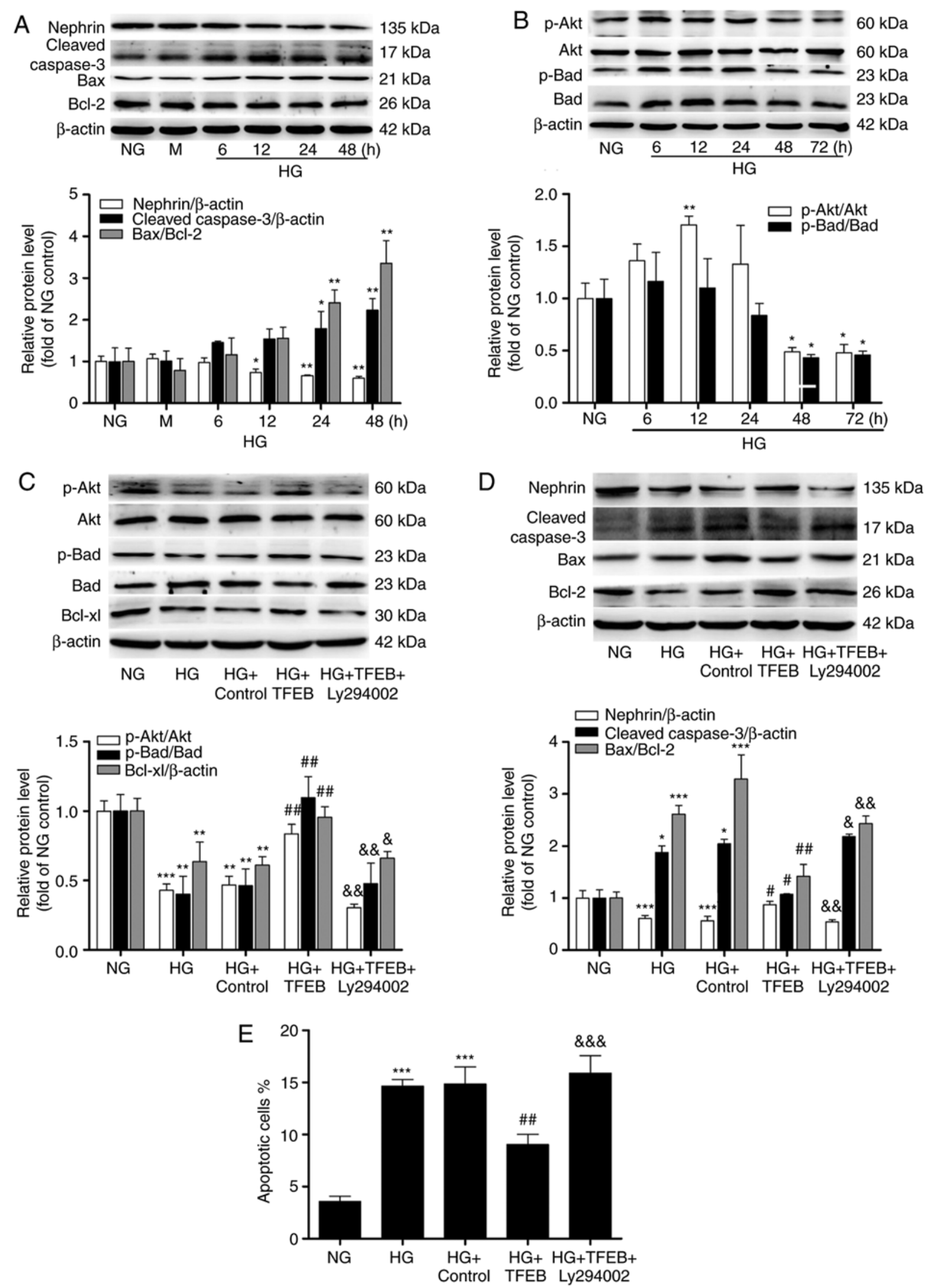

Figure 4. Effects of TFEB overexpression on podocyte apoptosis and the Akt/Bad pathway. (A) The expression levels of nephrin, cleaved-caspase-3 and $\mathrm{Bax} / \mathrm{Bcl}-2$ in podocytes exposed to $\mathrm{HG}$ at different time points were analyzed by western blot. (B) The expression levels of p-Akt, Akt, p-Bad, Bad and downstream Bcl-xl were detected by western blot. (C) p-Akt, AKT, p-Bad, Bad and Bcl-xl protein expression in the NG, HG, HG + Control, HG + TFEB and HG + TFEB + Ly294002 groups. (D) Nephrin, cleaved-caspase-3, Bax and Bcl-2 protein expression in the NG, HG, HG + Control, HG + TFEB and HG + TFEB + Ly294002 groups. (E) Flow cytometry was used to detect the apoptosis rate of each group when podocytes were exposed to HG for 48 h after

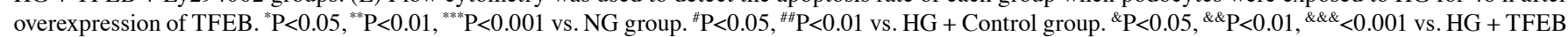
group. TFEB, transcription factor EB; HG, high glucose; p, phosphorylated; NG, normal glucose; M, mannitol-hypertonic control. 
Flow cytometry analyses demonstrated that the percentage of apoptotic cells in the HG group was significantly higher compared with that of the NG group (Figs. S2 and 4E). Overexpression of TFEB significantly reduced the HG-induced increase in apoptosis. The percentage of apoptotic cells in the HG + TFEB + Ly294002 group was significantly higher compared with that of the TFEB overexpression group; however, no significant difference was observed in comparison with the HG group (Figs. S2 and 4E).

\section{Discussion}

Previous studies have demonstrated that the expression of TFEB decreased in the myocardium of mice fed high-fat and high-sugar diets (27), and $\mathrm{HG}$ at $30 \mathrm{mmol} / 1$ could induce nuclear translocation of TFEB in U937 monocytic cells (28). Similarly, the present study identified that TFEB expression first increased and then decreased, and nuclear translocation occurred in mouse podocytes cultured in HG for different time points. The early increase may be due to a feedback mechanism that strengthens cells against undesirable factors and at the same time, entry into the nucleus initiates the transcription of downstream protective genes; however, when the stimulation persists, it causes the expression to decrease rapidly. It is hypothesized that TFEB may serve a positive role in diabetes and its complications.

ROS can induce the dephosphorylation of TFEB (29). When excessive ROS accumulate, oxidative stress occurs and triggers more pathophysiological cascades. Knockdown of TFEB can lead to overproduction of ROS in the mitochondria of osteoblasts, which can be attenuated by NAC (30), whereas overexpression of TFEB can remove excess ROS to a certain extent (31). The present study identified that $\mathrm{HG}$ resulted in a large accumulation of ROS in the cytoplasm and mitochondria of podocytes, and significantly reduced the expression levels of SOD2 and HO1. During overexpression of TFEB, oxidative stress was reduced and the expression levels of SOD2 and HO1 increased significantly, with no difference in stress level compared with the ROS scavenger NAC-treated group. RT-qPCR analysis demonstrated that the mRNA levels of SOD2 and HO1 increased by 5.7 and 3.5-fold, respectively, following overexpression of TFEB, which may be explained by the presence of TFEB-binding sites on SOD2 and HO1 gene promoters and introns (31). These results indicate that TFEB alleviates oxidative stress, reduces accumulated excess ROS and is associated with upregulation of the anti-oxidative stress proteins SOD2 and HO1.

TFEB has been reported to be involved in mitochondrial quality control, can improve the activity of respiratory chain complexes and can increase ATP synthesis $(17,32)$. The current results demonstrated that the TFEB-overexpression group exhibited significantly higher mRNA and protein levels of PGC-1 $\alpha$, TFAM and COX IV in podocytes compared with the HG group. PGC- $1 \alpha$ and TFAM are important regulators of mitochondrial biogenesis. Functionally, deacetylation of PGC- $1 \alpha$ activates TFAM, which in turn initiates the replication of mitochondrial DNA (33). COX IV is a key factor in COX assembly in the respiratory chain and is also a marker of mitochondria (34). Therefore, the current result suggests that TFEB enhances mitochondrial biogenesis. In addition, confocal microscopy demonstrated that the mitochondrial rod-like structure was destroyed and became swollen and fragmented after HG stimulation, whereas TFEB significantly improved the mitochondrial morphology. TFEB also partially restored the mitochondrial membrane potential that was reduced by HG. Considering the previously reported close association between TFEB and cellular autophagy/mitophagy (35), we hypothesize that TFEB serves an important role in protecting mitochondrial morphology and function, maintaining mitochondrial renewal, and maintaining mitochondrial homeostasis.

Renal intrinsic cell apoptosis is elevated in DN and in HG conditions in vitro $(36,37)$. The current study also demonstrated that in HG-induced apoptosis of podocytes, cleaved-caspase-3 and $\mathrm{Bax} / \mathrm{Bcl}-2$ were significantly increased. Mitochondria are the main targets of numerous pro-apoptotic factors and initiate apoptosis after injury. Akt/Bad is an apoptosis-inhibitory pathway involved in mitochondria (38). Animal studies have confirmed that by activating Akt/Bad, diabetes-induced apoptosis can be reduced (39). Following activation of Akt, Bad phosphorylates and binds to the 14-3-3 protein. This leads to dissociation of downstream Bcl-2 and Bcl-xl, which then bind to Bax to inhibit the pro-apoptotic effects of Bax $(40,41)$, blocking the cascade of subsequent apoptosis. The present study identified that phosphorylation of Akt and Bad decreased significantly after $48 \mathrm{~h}$ of $\mathrm{HG}$ stimulation and after $72 \mathrm{~h} \mathrm{p}$-Akt/Akt and p-Bad/Bad decreased to less than $50 \%$ of the NG group. TFEB can promote the phosphorylation of Akt (42). In the current study, overexpression of TFEB partially reversed the HG-reduced p-Akt/Akt and p-Bad/Bad, upregulated downstream Bcl-2 and Bcl-xl, decreased cleaved-caspase- 3 and increased the podocyte function protein nephrin. To further clarify whether TFEB could regulate the Akt pathway, Ly294002, an Akt phosphorylation inhibitor, was used. It was observed that the improvement by TFEB to apoptosis was significantly attenuated. In addition, flow cytometry for detecting the apoptosis rate in each group was consistent with this. Overall, the current data suggest that TFEB reduces HG-induced podocyte apoptosis by activating the Akt/Bad pathway to inhibit the mitochondrial apoptotic regulatory pathway.

The present findings suggest that overexpression of TFEB can reduce the production of ROS in podocytes in a HG environment, relieve oxidative stress, and promote mitochondrial biogenesis and renewal functions. Furthermore, TFEB could also reduce HG-induced podocyte apoptosis by activating the Akt/Bad pathway to inhibit the mitochondrial apoptotic regulatory pathway. Therefore, TFEB may be considered a potential therapeutic target for $\mathrm{DN}$.

However, there were certain limitations of the current study. Firstly, lack of information regarding TFEB location and the protein level in nuclei and cytoplasm at $72 \mathrm{~h}$ was a limitation. Due to the long time since the study, the data of the TFEB location and the protein level at $72 \mathrm{~h}$ cannot be supplemented. Other limitations include lack of design interaction experiments and lack of evaluation of the mitochondria ultrastructure, which need to be further investigated. In the future, attention should be paid to these issues to ensure the integrity of the experiments and data.

\section{Acknowledgements}

Not applicable. 


\section{Funding}

No funding was received.

\section{Availability of data and materials}

All data generated or analyzed during this study are included in this article.

\section{Authors' contributions}

All authors conceived and designed the experiments. YK and YL performed the experiments and analyzed the data. TZ wrote the manuscript. YC and ML modified the manuscript. All authors read and approved the final manuscript.

\section{Ethics approval and consent to participate}

The present study was approved by the Pathology Laboratory of Hebei Medical University (Shijiazhuang, China) for the use of purchased mouse podocytes. All procedures were performed in accordance with the World Medical Association's Declaration of Helsinki.

\section{Patient consent for publication}

Not applicable.

\section{Competing interests}

The authors declare that they have no competing interests.

\section{References}

1. Afkarian M, Sachs MC, Kestenbaum B, Hirsch IB, Tuttle KR, Himmelfarb J and de Boer IH: Kidney disease and increased mortality risk in type 2 diabetes. J Am Soc Nephrol 24: 302-308, 2013.

2. Collins AJ, Foley RN, Chavers B, Gilbertson D, Herzog C, Ishani A, Johansen K, Kasiske BL, Kutner N, Liu J, et al: US Renal Data System 2013 Annual Data Report. Am J Kidney Dis 63: A7, 2014.

3. Ogurtsova K, da Rocha Fernandes JD, Huang Y, Linnenkamp U, Guariguata L, Cho NH, Cavan D, Shaw JE and Makaroff LE: IDF Diabetes Atlas: Global estimates for the prevalence of diabetes for 2015 and 2040. Diabetes Res Clin Pract 128: 40-50, 2017.

4. Kawakami T, Ren S and Duffield JS: Wnt signalling in kidney diseases: Dual roles in renal injury and repair. J Pathol 229: 221-231, 2013.

5. Conti S, Perico N, Novelli R, Carrara C, Benigni A and Remuzzi G: Early and late scanning electron microscopy findings in diabetic kidney disease. Sci Rep 8: 4909, 2018.

6. Khazim K, Gorin Y, Cavaglieri RC, Abboud HE and Fanti P: The antioxidant silybin prevents high glucose-induced oxidative stress and podocyte injury in vitro and in vivo. Am J Physiol Renal Physiol 305: F691-F700, 2013.

7. Newsholme P, Haber EP, Hirabara SM, Rebelato EL, Procopio J, Morgan D, Oliveira-Emilio HC, Carpinelli AR and Curi R: Diabetes associated cell stress and dysfunction: Role of mitochondrial and non-mitochondrial ROS production and activity. J Physiol 583: 9-24, 2007.

8. Claussen AD, Fox DJ, Yu XC, Meech RP, Verhulst SJ, Hargrove TL and Campbell KC: D-methionine pre-loading reduces both noise-induced permanent threshold shift and outer hair cell loss in the chinchilla. Int J Audiol 52: 801-807, 2013.

9. Tsukagoshi H, Busch W and Benfey PN: Transcriptional regulation of ROS controls transition from proliferation to differentiation in the root. Cell 143: 606-616, 2010.
10. Fubini B and Hubbard A: Reactive oxygen species (ROS) and reactive nitrogen species (RNS) generation by silica in inflammation and fibrosis. Free Radic Biol Med 34: 1507-1516, 2003.

11. Yang HW, Hwang KJ, Kwon HC, Kim HS, Choi KW and Oh KS: Detection of reactive oxygen species (ROS) and apoptosis in human fragmented embryos. Hum Reprod 13: 998-1002, 1998.

12. Brownlee M: Biochemistry and molecular cell biology of diabetic complications. Nature 414: 813-820, 2001

13. Kim WH, Lee JW, Suh YH, Hong SH, Choi JS, Lim JH, Song JH, Gao B and Jung MH: Exposure to chronic high glucose induces beta-cell apoptosis through decreased interaction of glucokinase with mitochondria: Downregulation of glucokinase in pancreatic beta-cells. Diabetes 54: 2602-2611, 2005.

14. Steingrimsson E, Copeland NG and Jenkins NA: Melanocytes and the microphthalmia transcription factor network. Annu Rev Genet 38: 365-411, 2004.

15. Sardiello M, Palmieri M, di Ronza A, Medina DL, Valenza M, Gennarino VA, Di Malta C, Donaudy F, Embrione V, Polishchuk RS, et al: A gene network regulating lysosomal biogenesis and function. Science 325: 473-477, 2009.

16. Settembre C, Di Malta C, Polito VA, Garcia Arencibia M, Vetrini F, Erdin S, Erdin SU, Huynh T, Medina D, Colella P, et al: TFEB links autophagy to lysosomal biogenesis. Science 332: 1429-1433, 2011.

17. Ivankovic D, Chau KY, Schapira AH and Gegg ME: Mitochondrial and lysosomal biogenesis are activated following PINK1/parkin-mediated mitophagy. J Neurochem 136: 388-402, 2016.

18. Erlich AT, Brownlee DM, Beyfuss K and Hood DA: Exercise induces TFEB expression and activity in skeletal muscle in a PGC-1 $\alpha$-dependent manner. Am J Physiol Cell Physiol 314: C62-C72, 2018.

19. Martina JA, Diab HI, Brady OA and Puertollano R: TFEB and TFE3 are novel components of the integrated stress response. EMBO J 35: 479-495, 2016.

20. Pastore N, Brady OA, Diab HI, Martina JA, Sun L, Huynh T, Lim JA, Zare H, Raben N, Ballabio A and Puertollano R: TFEB and TFE 3 cooperate in the regulation of the innate immune response in activated macrophages. Autophagy 12: 1240-1258, 2016.

21. Peres GB, Schor N and Michelacci YM: Impact of high glucose and AGEs on cultured kidney-derived cells. Effects on cell viability, lysosomal enzymes and effectors of cell signaling pathways. Biochimie 135: 137-148, 2017.

22. Alghamdi TA, Majumder S, Thieme K, Batchu SN, White KE, Liu Y, Brijmohan AS, Bowskill BB, Advani SL, Woo M and Advani A: Janus kinase 2 regulates transcription factor EB expression and autophagy completion in glomerular podocytes. J Am Soc Nephrol 28: 2641-2653, 2017.

23. Covington MD and Schnellmann RG: Chronic high glucose downregulates mitochondrial calpain 10 and contributes to renal cell death and diabetes-induced renal injury. Kidney Int 81: 391-400, 2012.

24. Hou Y, Wu M, Wei J, Ren Y, Du C, Wu H, Li Y and Shi Y: CD36 is involved in high glucose-induced epithelial to mesenchymal transition in renal tubular epithelial cells. Biochem Biophys Res Commun 468: 281-286, 2015.

25. Ito Y, Hsu MF, Bettaieb A, Koike S, Mello A, Calvo-Rubio M, Villalba JM and Haj FG: Protein tyrosine phosphatase 1B deficiency in podocytes mitigates hyperglycemia-induced renal injury. Metabolism 76: 56-69, 2017.

26. Livak KJ and Schmittgen TD: Analysis of relative gene expression data using real-time quantitative PCR and the 2(-Delta Delta C(T)) Method. Methods 25: 402-408, 2001.

27. Trivedi PC, Bartlett JJ, Perez LJ, Brunt KR, Legare JF, Hassan A, Kienesberger PC and Pulinilkunnil T: Glucolipotoxicity diminishes cardiomyocyte TFEB and inhibits lysosomal autophagy during obesity and diabetes. Biochim Biophys Acta 1861: 1893-1910, 2016.

28. Tseng HHL, Vong CT, Kwan YW, Lee SM and Hoi MPM: Lysosomal $\mathrm{Ca}^{2+}$ signaling regulates high glucose-mediated interleukin-1 $\beta$ secretion via transcription factor EB in human monocytic cells. Front Immunol 8: 1161, 2017.

29. Zhang X, Cheng X, Yu L, Yang J, Calvo R, Patnaik S, Hu X, Gao Q, Yang M, Lawas M, et al: MCOLN1 is a ROS sensor in lysosomes that regulates autophagy. Nat Commun 7: 12109, 2016

30. Yoneshima E, Okamoto K, Sakai E, Nishishita K, Yoshida N and Tsukuba T: The transcription factor EB (TFEB) regulates osteoblast differentiation through ATF4/CHOP-dependent pathway. J Cell Physiol 231: 1321-1333, 2016. 
31. Lu H, Fan Y, Qiao C, Liang W, Hu W, Zhu T, Zhang J and Chen YE: TFEB inhibits endothelial cell inflammation and reduces atherosclerosis. Sci Signal 10, 2017.

32. Ma X, Liu H, Murphy JT, Foyil SR, Godar RJ, Abuirqeba H, Weinheimer CJ, Barger PM and Diwan A: Regulation of the transcription factor EB-PGC1 $\alpha$ axis by beclin-1 controls mitochondrial quality and cardiomyocyte death under stress. Mol Cell Biol 35: 956-976, 2015.

33. Aquilano K, Vigilanza P, Baldelli S, Pagliei B, Rotilio G and Ciriolo MR: Peroxisome proliferator-activated receptor gamma co-activator 1alpha (PGC-1alpha) and sirtuin 1 (SIRT1) reside in mitochondria: Possible direct function in mitochondrial biogenesis. J Biol Chem 285: 21590-21599, 2010.

34. Zsengellér ZK and Rosen S: The use of cytochrome C oxidase enzyme activity and immunohistochemistry in defining mitochondrial injury in kidney disease. J Histochem Cytochem 64: 546-555, 2016.

35. Nezich CL, Wang C, Fogel AI and Youle RJ: MiT/TFE transcription factors are activated during mitophagy downstream of Parkin and Atg5. J Cell Biol 210: 435-450, 2015.

36. Zhang X, Zhao Y, Chu Q, Wang ZY, Li H and Chi ZH: Zinc modulates high glucose-induced apoptosis by suppressing oxidative stress in renal tubular epithelial cells. Biol Trace Elem Res 158: 259-267, 2014

37. Zhang X, Liang D, Lian X, Jiang Y, He H, Liang W, Zhao Y and Chi ZH: Berberine activates Nrf2 nuclear translocation and inhibits apoptosis induced by high glucose in renal tubular epithelial cells through a phosphatidylinositol 3-kinase/Akt-dependent mechanism. Apoptosis 21: 721-736, 2016.
38. Zeng KW, Wang XM, Ko H, Kwon HC, Cha JW and Yang HO: Hyperoside protects primary rat cortical neurons from neurotoxicity induced by amyloid $\beta$-protein via the $\mathrm{PI} 3 \mathrm{~K} / \mathrm{Akt} / \mathrm{Bad} / \mathrm{Bcl}(\mathrm{XL})$-regulated mitochondrial apoptotic pathway. Eur J Pharmacol 672: 45-55, 2011.

39. Tao SC, Yuan T, Rui BY, Zhu ZZ, Guo SC and Zhang CQ: Exosomes derived from human platelet-rich plasma prevent apoptosis induced by glucocorticoid-associated endoplasmic reticulum stress in rat osteonecrosis of the femoral head via the $\mathrm{Akt} / \mathrm{Bad} / \mathrm{Bcl}-2$ signal pathway. Theranostics 7: 733-750, 2017.

40. Wang XM, Yao M, Liu SX, Hao J, Liu QJ and Gao F: Interplay between the Notch and PI3K/Akt pathways in high glucose-induced podocyte apoptosis. Am J Physiol Renal Physiol 306: F205-F213, 2014.

41. Nishihama K, Yasuma T, Yano Y, D' Alessandro-Gabazza CN, Toda M, Hinneh JA, Baffour Tonto P, Takeshita A, Totoki T, Mifuji-Moroka R, et al: Anti-apoptotic activity of human matrix metalloproteinase-2 attenuates diabetes mellitus. Metabolism 82: 88-99, 2018.

42. Mansueto G, Armani A, Viscomi C, D'Orsi L, De Cegli R, Polishchuk EV, Lamperti C, Di Meo I, Romanello V, Marchet S, et al: Transcription factor EB controls metabolic flexibility during exercise. Cell Metab 25: 182-196, 2017.

This work is licensed under a Creative Commons

Attribution-NonCommercial-NoDerivatives 4.0 International (CC BY-NC-ND 4.0) License. 